\title{
Involvement of the Periaqueductal Gray in the Descending Antinociceptive Effect Induced by the Central Nucleus of Amygdala
}

\author{
N. BOURBIA ${ }^{1,2}$, A. PERTOVAARA ${ }^{1}$ \\ ${ }^{1}$ Department of Physiology, Faculty of Medicine, University of Helsinki, Helsinki, Finland, ${ }^{2}$ MRC \\ Harwell Institute, Oxfordshire, United Kingdom
}

Received June 25, 2017

Accepted March 2, 2018

On-line May 10, 2018

\section{Summary}

Here we studied whether descending control of mechanical nociception by glutamate in the central nucleus of the amygdala (CeA) of healthy control animals is induced by amygdaloid NMDA receptors and relayed through the midbrain periaqueductal gray (PAG). Mechanical nociception in the hind paws was assessed in rats with chronic guide cannulae for glutamate administration in the right $\mathrm{CeA}$ and for inducing local anesthesia in the PAG. In a separate electrophysiological study, ON-like PAG neurons giving an excitatory response to noxious pinch of the tail were recorded in anesthetized rats following glutamate administration into the CeA. A high dose of glutamate $(100 \mu \mathrm{g})$ in the CeA induced mechanical antinociception in the contra- but not ipsilateral hind limb. Antinociception was prevented by an NMDA receptor antagonist in the CeA or local anesthesia of the PAG. Discharge rate of ON-like PAG neurons was increased by a high dose of glutamate $(100 \mu \mathrm{g})$ in the CeA and this increase was prevented by an NMDA receptor antagonist in the CeA. The results indicate that amygdaloid NMDA receptors in the CeA may induce contralaterally mechanical antinociception through a circuitry relaying in the PAG. Activation of ON-like PAG neurons is associated with the descending antinociceptive effect. Mechanisms and causality of this association still remain to be studied.

\section{Key words}

Amygdala • Descending pain control • Mechanical nociception • $\mathrm{N}$-methyl-D-aspartate receptor $\bullet$ Periaqueductal gray

\section{Corresponding author}

A. Pertovaara, Department of Physiology, Faculty of Medicine, University of Helsinki, POB 63, 00014 Helsinki, Finland. E-mail: antti.pertovaara@helsinki.fi

\section{Introduction}

Central nucleus of the amygdala (CeA), the main output nucleus of the amygdala, is involved in processing of primary emotions, such as fear (Le Doux 2007), but among its many functions is processing and regulation of pain- and nociception-related responses (Neugebauer et al. 2009). Concerning descending regulation of nociception, $\mathrm{CeA}$ has efferent projections to brainstem structures involved in descending control of nociception. Among them are the midbrain periaqueductal gray (PAG) (Da Costa Gomez and Behbehani 1995, Oka et al. 2008, Rizvi et al. 1991) and the pontine locus coeruleus (LC) (Van Bockstaele et al. 2001). It has been reported that attenuation of spinal heat nociception induced by electric stimulation of the $\mathrm{CeA}$ was abolished following local anesthesia of the PAG (Oliveira and Prado 2001) indicating that the $\mathrm{CeA}$ projection to the $\mathrm{PAG}$ rather than e.g. the LC relayed the descending antinociceptive effect. Descending control mechanisms of spinal nociception may vary with submodality of test stimulation as indicated by the finding that transection of the spinal cord had opposite effects on mechanical and heat nociception (Kauppila et al. 1998). Therefore, the earlier finding that blocking the PAG abolished the CeA-induced heat antinociception (Oliveira and Prado 2001) does not exclude the possibility that the CeA-induced descending control of mechanical nociception is relayed by brainstem structures other than the PAG.

Recently we showed that a high dose of glutamate in the CeA suppresses mechanical nociception in the contralateral limb and that this descending 
antinociceptive effect was reversed by pretreatment of the CeA with an NMDA receptor antagonist (Bourbia et al. 2014). In the present study, we determined whether this amygdaloid NMDA receptor-induced mechanical antinociceptive effect is relayed by the PAG. Therefore, we assessed whether local anesthesia of the PAG abolishes the mechanical antinociceptive effect induced by NMDA receptor-mediated action of glutamate the CeA. Moreover, the response of nociceptive PAG neurons to amygdaloid glutamate was determined to have a neuronal correlate of a possible relay mechanism.

\section{Materials and Methods}

Animals

Adult male Hannover-Wistar rats (Envigo, Horst, The Netherlands), weighing 200-350 g, were used to perform the experiments. The Experimental Animal Ethics Committee of the Provincial Government of Southern Finland (Hämeenlinna, Finland) approved the study protocol (permission \# ESAVI/7863/04.10.07/2013), and the experiments were performed according to the guidelines of European Communities Council Directive of 22 September 2010 (2010/63/EU). All efforts were made to limit distress and to use only the number of animals necessary to produce reliable scientific data. Rats were housed in a 12-h light/dark cycle with food and water access ad libitum.

\section{Guide cannula insertion and drug injection procedure}

The animals had a guide cannula for drug administration into the right $\mathrm{CeA}$ as described in detail earlier (Bourbia et al. 2010). The right CeA was chosen as a target in this study, since previous experimental animals studies indicate that the right $\mathrm{CeA}$ has a more important role in pain processing than the left $\mathrm{CeA}$ (Carrasquillo and Gereau 2008, Ji and Neugebauer 2009, Kolber et al. 2010), although not in all conditions (Spuz and Borszcz 2012). For placement of the guide cannulae (26 gauge; PlasticsOne, Roanoke, VA, USA), the animal was placed into a standard stereotaxic frame, skull was exposed and two holes drilled for their placement under pentobarbitone anesthesia $(60 \mathrm{ml} / \mathrm{kg}$ i.p.). One of the guide cannulae was placed in the right CeA: $6.8 \mathrm{~mm}$ anterior from the interaural line, $4.2 \mathrm{~mm}$ lateral from the midline, and $7.9 \mathrm{~mm}$ ventral from the dura mater (Paxinos and Watson 1982). The other guide cannula was placed into the PAG: $2.0 \mathrm{~mm}$ anterior from the interaural line, $0.8 \mathrm{~mm}$ lateral from the midline, and 5-6 mm ventral from the dura mater. The guide cannulae were kept in place with dental cement and two dental screws inserted in the skull bone. For treatment of postoperative pain, the animals were given buprenorphine at a dose of $0.01 \mathrm{mg} / \mathrm{kg}$ intramuscularly starting during the operation and continuing twice daily for three days postoperatively. The rats had one week time to recover from the operation before starting the behavioral experiment. Effect of glutamate in a brain control injection site was not assessed in the present experiment, since the same experimenters using identical methods showed earlier that glutamate at the currently used dose in the right internal capsule does not influence mechanical nociception in healthy control rats, unlike it did in the right CeA (Bourbia et al. 2014).

Intracerebral injections of drugs or saline were performed at the volume of $0.5 \mu \mathrm{l}$ by using 30-gauge injection (PlasticsOne) needle connected to a $10-\mu \mathrm{l}$ Hamilton microsyringe (Hamilton Company, Bonaduz, Switzerland) by polyethylene (PE-20) tubing. The injection needle protruded $2.0 \mathrm{~mm}$ beyond the cannula tips. The animals were gently restrained during the injection procedure. The injection lasted $20 \mathrm{~s}$ after which the injection needle was kept in place for another $20 \mathrm{~s}$ to maximize diffusion and to prevent back flow of the drug into the cannula.

\section{Assessment of limb-withdrawal threshold to mechanical stimulation}

Rats were habituated three days to the experimental conditions. For assessment of mechanical nociception, the limb withdrawal threshold was determined by mechanical stimulation of the right and left hind paw with calibrated monofilaments producing forces that varied from $0.008 \mathrm{~g}$ to $300 \mathrm{~g}$. Testing was performed while the animal was standing on a metal grid through which the monofilaments were applied to the plantar skin of the hind paw.

The experimental test consists in five consecutive stimulations of the foot pads with monofilaments at an ascending series of force and at a stimulation frequency of $1 \mathrm{~Hz}$. The lowest filament force producing a $100 \%$ withdrawal rate of the limb (i.e. the lowest force evoking a limb withdrawal every time it was presented) was considered as the withdrawal threshold.

\section{Course of the behavioral study}

Experiments were performed 1-2 weeks after 
cannula insertion. Mechanical nociception was tested in the following drug conditions: saline or lidocaine (4\%) in the PAG followed $5 \mathrm{~min}$ later by one of the following treatments of the CeA: saline alone, glutamate alone at the dose of $100 \mu \mathrm{g}, \mathrm{MK}-801$ alone at the dose of $2 \mu \mathrm{g}$, or MK-801 at the dose of $2 \mu \mathrm{g}$ followed 5 min later by glutamate at the dose of $100 \mu \mathrm{g}$. The order of testing these different drug treatment conditions was randomized using QuickCalcs software (www.graphpad.com). Withdrawal threshold determination started $1 \mathrm{~min}$ following completion of the drug treatment procedure. The minimum interval in testing different drug conditions in the same animal was 1 day.

\section{Electrophysiological study}

Electrophysiological recordings of PAG neurons were performed in a separate experiment. Electrophysiological recording was performed under sodium pentobarbitone anesthesia at a dose of $60 \mathrm{mg} / \mathrm{kg}$ i.p. for the induction of anesthesia, then followed by administering $15-20 \mathrm{mg} / \mathrm{kg} / \mathrm{h}$ or more, when required to maintain rats under stable anesthesia. The anesthetized animals were placed in a standard stereotaxic frame. The skull was exposed and two holes were drilled in the skull: one for the recording electrode to be inserted into the PAG $(2 \mathrm{~mm}$ anterior from the interaural line, $0.8 \mathrm{~mm}$ lateral from the midline, 4.6-6.2 $\mathrm{mm}$ ventral from the dura mater) and the other hole for the guide cannula insertion into the $\mathrm{CeA}(6.8 \mathrm{~mm}$ anterior from the interaural line, $4.2 \mathrm{~mm}$ lateral from the midline, $7.9 \mathrm{~mm}$ ventral from the dura mater) according to the coordinates of the rat stereotaxic atlas of Paxinos and Watson (1982).

The level of the anesthesia was frequently assessed by pinching the paws and ears, and supplemental doses of the anesthetic were given as needed. While the level of anesthesia may vary in spite of attempts to maintain anesthesia at a constant level, it is important to note that anesthesia was maintained in an identical manner in all experimental conditions. This was expected to minimize the contribution of the potential differences in anesthesia level to the differences in results between the experimental conditions within this study. The rats were spontaneously breathing. A warming blanket was used to maintain body temperature within physiological range.

A lacquer-coated tungsten electrode (tip impedance 3-10 $\mathrm{M} \Omega$ at $1 \mathrm{kHz}$; FHC Inc., Bowdoinham, ME, USA) was used to record extracellularly single neuron activity which was amplified and filtered using standard methods. A computer connected to a CED Micro 1401 interface and using Spike 2 software (Cambridge Electronic Design, Cambridge, UK) was used to perform the data sampling. Spike 2 software classifies waveform shapes based on full wave templating, and in the offline analysis the template matching can be complemented by clustering using principal component analysis. PAG neurons were classified according their response to an innocuous brush of the hind paw with a camel hair brush and a noxious pinch of the tail with a hemostatic clamp that produced pain when applied to the experimenter's hand. Only neurons with an excitatory response to noxious pinch were studied further. An earlier study described a neuron type called ON-cell in the PAG that gave an excitatory response to noxious heat prior to the heat-evoked spinal withdrawal reflex (Heinricher et al. 1987). The PAG neurons recorded in the present study resembled those classified as ON-cells of the rostroventromedial medulla in earlier studies (Fields et al. 1991). However, since in the present study withdrawal reflex was not assessed in parallel with the neuronal response and since the submodality of noxious test stimulation was different (pinch, instead of heat), the neurons recorded in the present study were called ON-like neurons. It should be noted that the use of a pinch for classification of PAG neurons was likely to lead to a bias in types of PAG neurons sampled. Namely, when the ongoing discharge of the PAG neuron is low or non-existent, it may not be possible to detect OFF-like PAG neurons giving an inhibitory response to pinch.

\section{Course of the electrophysiological study}

After finding a PAG neuron that gave an excitatory response to noxious pinch of the tail, its spontaneous activity was recorded for at least $1 \mathrm{~min}$ before drug treatments. One of the drug treatment conditions was administration of saline followed $5 \mathrm{~min}$ later by glutamate at the dose of $100 \mu \mathrm{g}$. The other drug treatment condition was administration of MK-801 at the dose of $2 \mu \mathrm{g}$ followed $5 \mathrm{~min}$ later by administration of glutamate at the dose of $100 \mu \mathrm{g}$. Neuronal discharge was recorded up to $15 \mathrm{~min}$ after drug administrations. The interval between testing the different drug treatment conditions was at least $60 \mathrm{~min}$. The drug doses were chosen based on results of our previous study, in which glutamate at the dose of $100 \mu \mathrm{g}$ in the CeA of healthy animals had a mechanical antinociceptive effect that was prevented by pretreating the CeA with MK-801 at the 
dose of $2 \mu \mathrm{g}$ (Bourbia et al. 2014). The order of testing different drug treatment conditions was varied randomly. After completing the recording, an electrolytic lesion was made by applying $50 \mu \mathrm{A}$ direct current for $30 \mathrm{~s}$ in the recording site.

\section{Drugs}

Glutamate and MK-801 (an NMDA receptor antagonist) were purchased from Sigma-Aldrich (St. Louis, MO, USA) and lidocaine (4\%) from Astra-Zeneca (Södertälje, Sweden). Physiological saline (OrionPharma, Espoo, Finland) was used for control injections.

\section{Histology}

After completion of the experiments, rats were sacrificed by giving them a lethal dose of sodium pentobarbitone and the brain was immediately removed and placed in $10 \%$ formaldehyde. Coronal sections of the brain were made to verify recording and injection sites in the brain. A schematic representation of the sites of cannula placements in the right $\mathrm{CeA}$ as well as the cannula and recording electrode placements in the PAG are shown in Figure 1.

\section{Statistical analyses}

Behavioral data were analyzed using nonparametric Friedman's test followed by Dunn's test or when comparing two conditions, Wilcoxon's test. Neuronal data were analyzed using repeated-measures one-way ANOVA followed by Tukey's test. Statistical analyses were performed with the GraphPad Prism 5 software (GraphPad Inc., La Jolla, CA, USA). P<0.05 was considered to represent a significant difference.

\section{Results}

\section{Behavioral study}

Spinal antinociception induced by glutamate in the right $C e A$ is prevented by blocking the $P A G$

Drug treatments of the present study did not produce obvious side-effects, such as motor paralysis, vocalizations, freezing or escape oriented behavior. Drug treatments of the right CeA and PAG had a significant effect on withdrawal threshold in the contralateral hind limb $(F r=14.86, P<0.0001$; Fig. 2A). Following pretreatment of the right $\mathrm{PAG}$ with saline, a high dose of glutamate $(100 \mu \mathrm{g})$ in the right $\mathrm{CeA}$ induced an increase of the mechanical withdrawal threshold in the contralateral hind limb (Fig. 2A), but failed to influence the threshold of the ipsilateral hind limb (Fig. 2B).

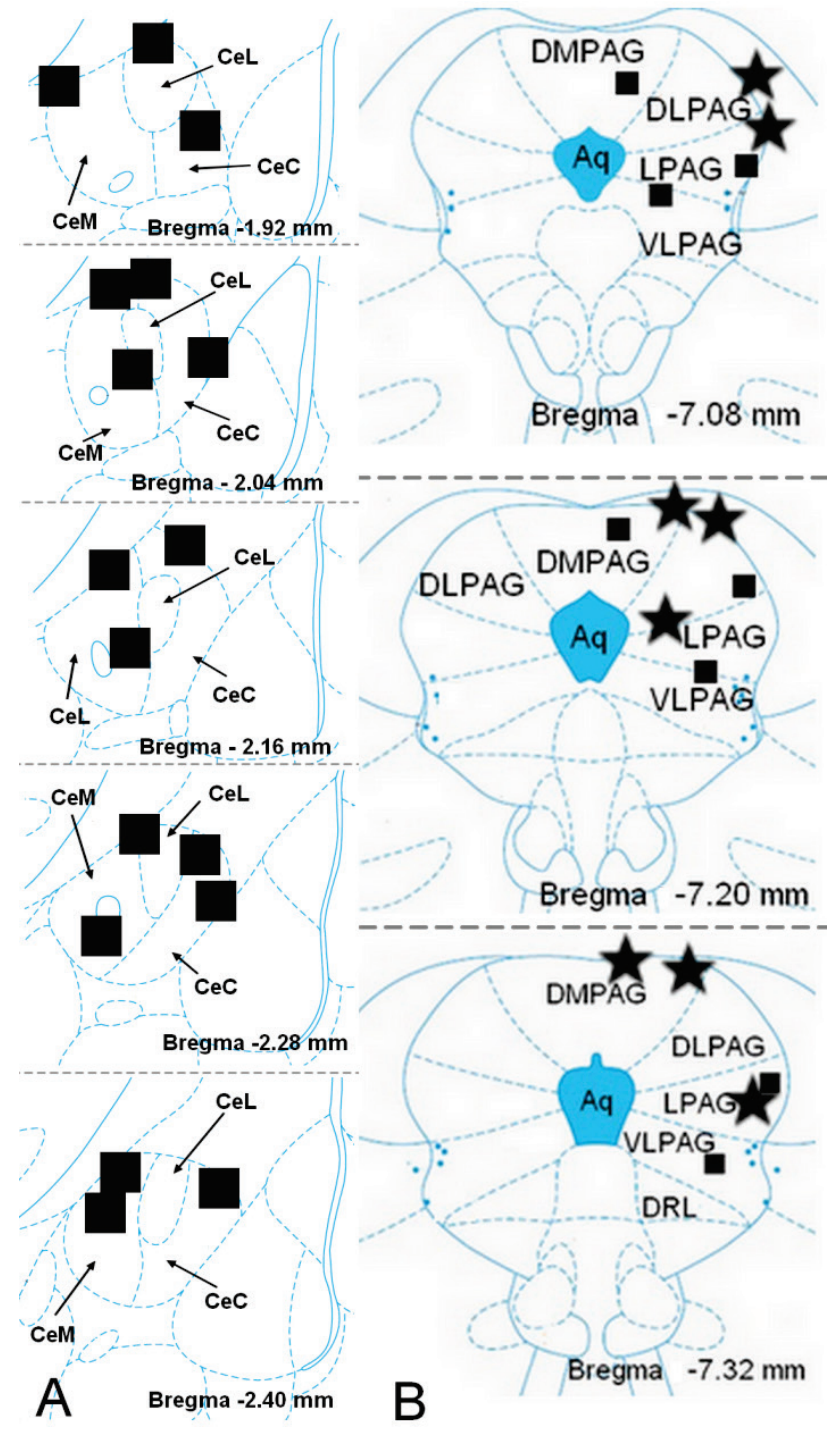

Fig. 1. A. Schematic representation of the sites of cannula placements (black squares) in the right central nucleus of amygdala. $\mathrm{CeC}$, Capsular nucleus of the central amygdala; $\mathrm{CeM}$, Medial division of the central nucleus of amygdala; CeL, Lateral division of the central nucleus of amygdala. B. Verified recording electrode sites (black squares) and injection cannula sites (black stars) in the periaqueductal gray. Each black square represents one electrode which has recorded between 1 to 3 neurones. Aq, aqueduct; DMPAG, Dorsomedial periaqueductal gray; DLPAG, Dorsolateral periaqueductal gray; LPAG, Lateral periaqueductal gray; VLPAG, Ventrolateral periaqueductal gray; $\mathrm{DRL}$, Lateral part of the dorsal raphe nucleus.

Pretreatment of the PAG with a local anesthetic (lidocaine $4 \%$ ) prevented the increase of the mechanically evoked withdrawal threshold in the contralateral limb induced by a high dose of glutamate $(100 \mu \mathrm{g})$ in the right CeA (Fig. 2C). After local anesthesia of the PAG, the contralateral withdrawal threshold was not different between animals receiving saline or $100 \mu \mathrm{g}$ of glutamate in the right $\mathrm{CeA}$ (Fig. 2C). None of the three drug administration conditions influenced the threshold of the ipsilateral hind limb $(F r=3.39, P=0.15$; Fig. 2D). 

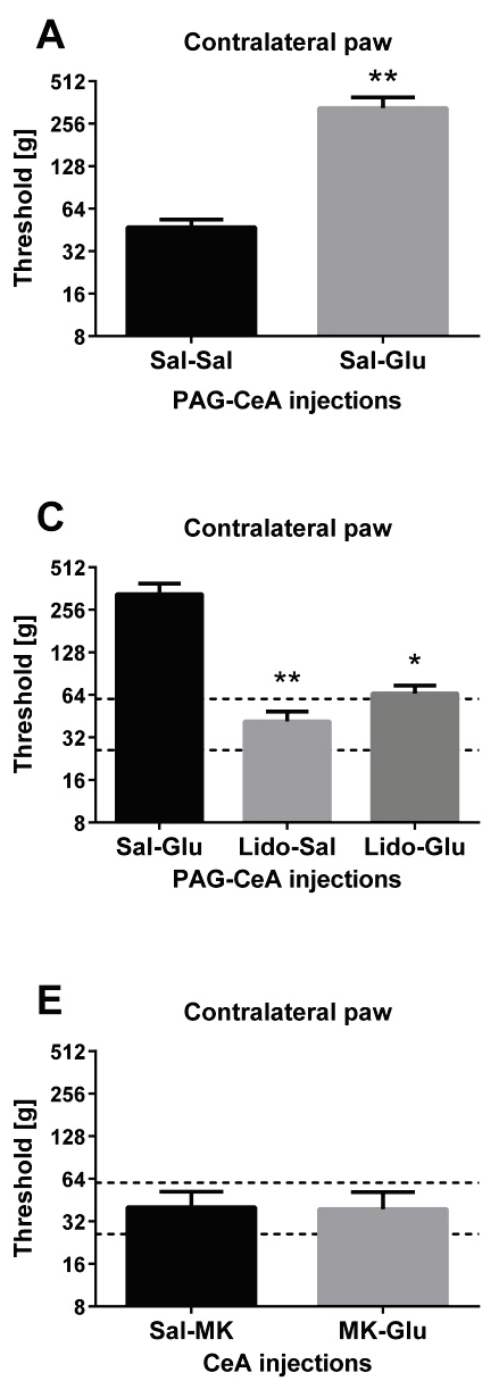
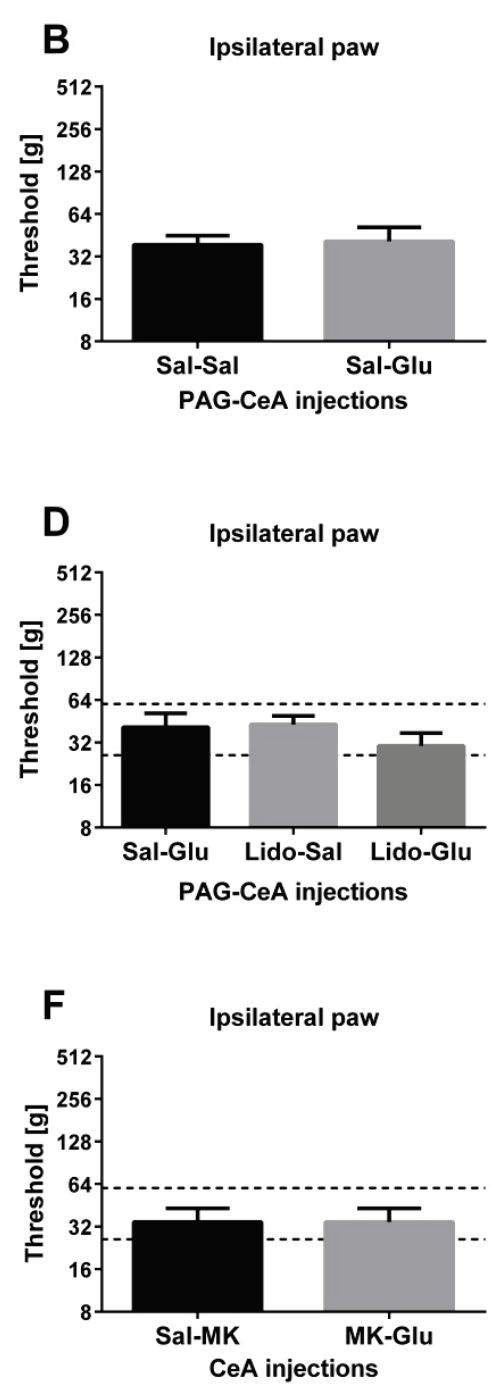

Fig. 2. Mechanical withdrawal thresholds in the hind $\operatorname{limb}$ contralateral $(\mathbf{A}, \mathbf{C}, \mathbf{E})$ and ipsilateral (B, D, E) to the right central nucleus of the amygdala (CeA) and periaqueductal gray (PAG) into which drugs were microinjected. Treatment conditions were saline (Sal) in the PAG followed by saline or glutamate (Glu, $100 \mu \mathrm{g}$ ) in the CeA (A and B), saline or lidocaine (Lido, $4 \%$ ) in the PAG followed by saline or glutamate in the CeA (C and D), and saline or MK-801 (MK, $2 \mu \mathrm{g})$ in the CeA followed saline or glutamate in the CeA (E and $\mathbf{F})$. Broken horizontal lines represent the interquartile range of the median threshold in the corresponding saline-treated control group: error bars represent SEM $(n=8$ in graphs $A-D$, and $n=4$ in graphs $E$ and $F$ ) ${ }^{*} \mathrm{P}<0.05,{ }^{* *} \mathrm{P}<0.01$ (re: the Sal-Glu group; Willcoxon's test in A, and Dunn's test in C).
Following pretreatment of the right $\mathrm{CeA}$ with $2 \mu \mathrm{g}$ of MK-801 (an NMDA receptor antagonist), $100 \mu \mathrm{g}$ of glutamate in the $\mathrm{CeA}$ failed to induce an increase of the hind limb withdrawal threshold in the contralateral (Fig. 2E) or the ipsilateral (Fig. 2F) hind limb (Fig. 2E). Following treatment of the right $\mathrm{CeA}$ with $2 \mu \mathrm{g}$ of MK-801 alone, the contralateral (Fig. 2E) and ipsilateral (Fig. 2F) hind limb withdrawal thresholds were within the interquartile range of the corresponding median threshold in saline-treated animals (Fig. 2E).

\section{Electrophysiological study}

Glutamate acting on $N M D A$ receptors in the right CeA increases discharge of ON-like neurons in the PAG

Ten PAG neurons responding to noxious pinch of the tail were recorded. Eight of them gave an excitatory response to pinch and were classified as ON-like neurons, while two neurons gave an inhibitory response to pinch and were classified as OFF-like neurons. Here, only ON-like neurons were studied further. The mean spontaneous discharge rate of ON-like neurons was $0.5 \pm 0.3 \mathrm{~Hz}$ ( \pm SEM, $\mathrm{n}=8)$. The mean discharge rate was $0.9 \pm 0.4 \mathrm{~Hz}$ during stimulation with an innocuous brush and $5.8 \pm 1.3 \mathrm{~Hz}$ during noxious pinch of the tail. The after-discharge following the end of the noxious tail pinch of $5 \mathrm{~s}$ duration lasted $20.6 \pm 6.0 \mathrm{~s}$.

Six PAG ON-like neurons that were in the ventrolateral or lateral $\mathrm{PAG}$ were studied in all $\mathrm{CeA}$ treatment conditions (saline, glutamate, and MK-801 followed by glutamate). Figure 3 shows an example of the response of an ON-like neuron of the PAG to drug treatments of the right $\mathrm{CeA}$ : saline failed to influence the discharge rate of the PAG neuron, whereas $100 \mu \mathrm{g}$ of glutamate induced a short-lasting increase of the ongoing discharge rate that was prevented by pretreatment of the CeA with MK-801 at a dose of $2 \mu \mathrm{g}$ that alone failed to influence the discharge rate of the PAG neuron. Before drug treatment, the studied ON-like neuron had no spontaneous activity and gave an excitatory response to noxious pinch of the tail but not to innocuous brushing 
(lowest trace in Fig. 3). Overall studied neurons, drug treatments of the right $\mathrm{CeA}$ significantly influenced the discharge rate of ON-like neurons $\left(F_{1,5}=8.95, P=0.028\right.$; Fig. 4). Post hoc tests indicated that when compared with the effect of saline, the increase of the glutamate-induced increase of the discharge rate was significant, except in the condition in which $\mathrm{CeA}$ was pretreated with $2 \mu \mathrm{g}$ of MK-801 before administration of glutamate (Fig. 4).

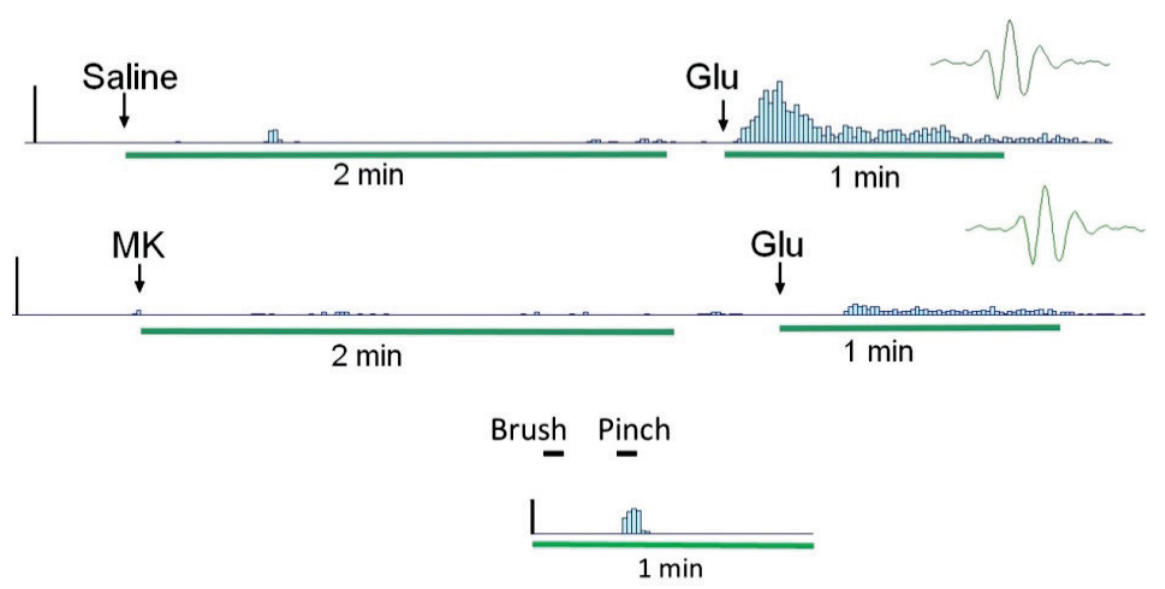

Fig. 3. Examples of the response of a single ON-like neuron in the periaqueductal gray (PAG) following microinjections of drugs into the right central nucleus of the amygdala (CeA). The uppermost row shows that saline failed to influence the discharge rate whereas $100 \mu \mathrm{g}$ of glutamate induced a short-lasting increase in the discharge rate. The middle row shows that MK-801 at the dose of $2 \mu \mathrm{g}$ failed to influence the discharge rate, while it prevented the glutamate-induced increase of the discharge rate. The lowermost row shows that prior to drug administrations, the PAG neuron was activated by noxious pinching of the tail but not by innocuous brushing. The insets show the shape of the action potential in the studied PAG neuron and the vertical calibration bars represent $50 \mathrm{~Hz}$.

PAG ON-like neurons

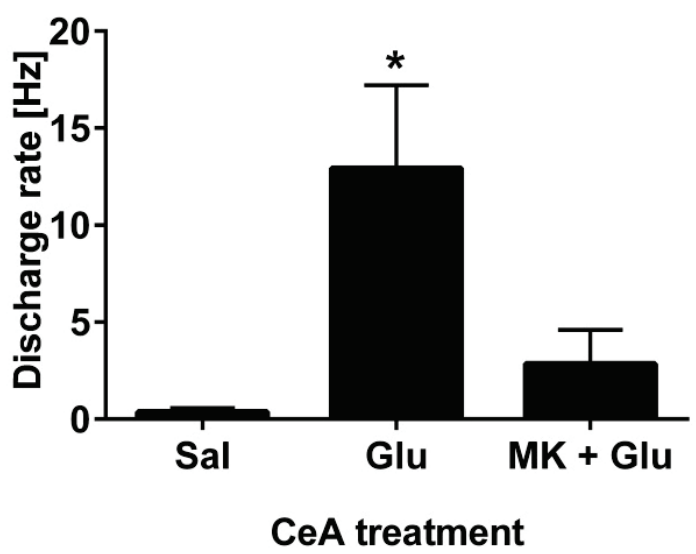

Fig. 4. Mean responses of the ON-like PAG neurons to microinjections of drugs into the right CeA. Sal, saline; Glu, glutamate $(100 \mu \mathrm{g}) ;$ MK+Glu, MK-801 $(2 \mu \mathrm{g})$ followed 2 min later by $100 \mu \mathrm{g}$ of glutamate. Error bars represent SEM $(n=6)$. $* \mathrm{P}<0.05$ (reference: the saline group; Tukey's test).

\section{Discussion}

It has been shown earlier that heat antinociception induced by electrical stimulation of the $\mathrm{CeA}$ is relayed downwards through the PAG (Oliveira and Prado 2001). The present results extend this earlier finding by showing that the mechanical antinociceptive effect induced by a high dose of glutamate in the CeA is also relayed downwards through the PAG, as shown by reversal of the antinociceptive effect following local anesthesia of the PAG. The behavioral antinociceptive effect induced by glutamate in the $\mathrm{CeA}$ was accompanied by an increase in the discharge rate of ON-like PAG neurons. Both the CeA-induced behavioral antinociceptive effect and the accompanying increase of the discharge rate of ON-like PAG cells were reversed by blocking amygdaloid NMDA receptors. Together, these findings suggest that amygdaloid NMDA receptors and ON-like PAG neurons are involved in the descending mechanical antinociceptive effect induced by a high dose of glutamate in the CeA.

Earlier studies have shown that stimulation of the dorsolateral or lateral PAG produces "fight" or "flight" responses, increased blood pressure and heart rate and a brief nonopioid-mediated antinociception, whereas stimulation of the ventrolateral PAG may produce freezing, decreased reactivity to the environment, and a longer-term, opioid-mediated antinociception (Lovick 1993). In the present study, half of the ON-like PAG neurons studied in all drug conditions were in the ventrolateral subregion, the stimulation of which produces powerful antinociception (Fardin et al. 1984), whereas the other half were in the adjacent lateral subregion, the stimulation of which has produced weaker and shorter-lasting antinociception (Lovick 1993). In the present study, the responses of 
ON-like PAG neurons induced by glutamate in the CeA did not vary with the PAG subregion, although it should be emphasized that the sample size was small. On the other hand, lidocaine-induced block of PAG was in all animals in the dorsolateral or lateral subregion leaving out a possibility that blocking ventrolateral subregion might have had an effect different from that described in the present study.

Interestingly, while activation of $\mathrm{ON}$-cells in the rostroventromedial medulla, an important relay between the PAG and the spinal dorsal horn, has been associated with pro- rather than antinociceptive actions (Fields et al. 1991), activation of ON-like PAG cells was associated with mechanical antinociception in the present study. Earlier studies have demonstrated that descending antinociception induced by stimulation of the PAG is relayed through the RVM (Gebhart et al. 1983). Due to complexity of the circuitry relaying descending pain control in the PAG (Lau and Vaughan 2014), one needs to be cautious with the interpretation of the present neuronal findings. This caveat is relevant to keep in mind particularly since it still remains to be studied whether the CeA glutamate-activated ON-like PAG cells were releasing excitatory (e.g. glutamate) or inhibitory (GABA) neurotransmitters and whether they had axonal projections only within the PAG or e.g. to the RVM. Among potential explanations for the present findings is that direct or indirect descending projections of ON-like PAG cells drive antinociceptive OFF-cells and/or suppress pronociceptive ON-cells in the RVM.

It should be noted that the present study was performed in healthy control animals. In neuropathic animals, unlike in healthy controls, blocking the amygdaloid NMDA receptors with a dose of MK-801 that alone had no effect on spinal nociception failed to reverse the descending antinociceptive effect induced by a high dose of glutamate in the CeA (Sagalajev et al. 2015). However, MK-801 at a dose producing no change in pain behavior of neuropathic animals when administered alone reversed the pronociceptive effect induced by a low dose of glutamate in the CeA (Sagalajev et al. 2015). This finding suggests that the role of amygdaloid NMDA receptors in descending control of nociception in healthy controls differs from that in neuropathy. On the other hand, the dose of MK-801 that alone was ineffective in neuropathic animals of our earlier study (Sagalajev et al. 2015) was an order of magnitude lower than the corresponding dose in healthy controls of the present study $(0.1 \mu \mathrm{g}$ versus $2.0 \mu \mathrm{g})$.
Therefore, we cannot exclude the possibility that blocking the amygdaloid NMDA receptors with a high dose of MK-801 had reduced the descending antinociceptive effect induced by a high dose of glutamate in neuropathic animals of our earlier study as well as in control animals of the present study. Another noteworthy difference in the pain control by the CeA between neuropathic and healthy control animals was shown in our recent behavioral study demonstrating that local anesthesia of the $\mathrm{CeA}$ attenuated pain hypersensitivity in neuropathic animals but failed to influence nociception in healthy controls (Sagalajev et al. 2018). This finding suggests that the CeA or nerve fibers passing through it have a tonic pain facilitatory effect in neuropathy but no tonic net effect on descending pain control under physiological conditions.

The role of amygdaloid NMDA receptors in pain processing may vary depending on whether it is involved in mediating ascending nociceptive signals or descending controls and on the pathophysiological condition. This is indicated by the present finding that amygdaloid NMDA receptors induced a descending antinociceptive effect in healthy controls of the present study while earlier studies indicate that the descending pronociceptive effect induced by the $\mathrm{CeA}$ of neuropathic animals was at least partly mediated by amygdaloid NMDA receptors (Ansah et al. 2010, Sagalajev et al. 2015) and that peripheral neuropathy-induced synaptic plasticity leading to amplification of ascending nociceptive signals to the CeA was partly dependent on amygdaloid NMDA receptors in arthritic animals ( $\mathrm{Li}$ and Neugebauer 2004). In neuropathic animals, however, amplification of ascending nociceptive signals from the parabrachial nucleus to the CeA has been independent of amygdaloid NMDA receptors (Ikeda et al. 2007).

Among limitations of the present study is that the focus was on nociceptive thresholds which represent sensory-discriminative aspects of pain. Amygdala, its NMDA receptors and projections to the PAG have been shown to contribute also to the control of pain affect (Spuz et al. 2014). Moreover, it should be noted that various subtypes of metabotropic glutamate receptors that were not studied here are also involved in pain control both in the CeA and the PAG (Palazzo et al. 2014).

\section{Conclusions}

The circuitry mediating the mechanical antinociceptive effect induced by a high dose of 
glutamate in the CeA of healthy rats involves amygdaloid NMDA receptors. Moreover, the antinociceptive effect was associated with activation of ON-like cells in the midbrain PAG. However, the underlying mechanisms and the potential causality of the association of the CeA glutamate-induced activation of ON-like PAG cells with behavioral antinociception still remains to be studied.

\section{Conflict of Interest}

There is no conflict of interest.

\section{Acknowledgements}

The authors were supported by grants from the Yrjö and Tuulikki Ilvonen Foundation, the Emil Aaltonen Foundation, the Finnish Cultural Foundation, and the Sigrid Jusélius Foundation.

\section{References}

ANSAH OB, BOURBIA N, GONÇALVES L, ALMEIDA A, PERTOVAARA A: Influence of amygdaloid glutamatergic receptors on sensory and emotional pain-related behavior in the neuropathic rat. Behav Brain Res 209: 174-178, 2010.

BOURBIA N, ANSAH OB, PERTOVAARA A: Corticotropin-releasing factor in the rat amygdala differentially influences sensory-discriminative and emotional-like pain response in peripheral neuropathy. J Pain 11: 1461-1471, 2010.

BOURBIA N, SAGALAJEV B, PERTOVAARA A: Descending effect on spinal nociception by amygdaloid glutamate varies with the submodality of noxious test stimulation. Neurosci Lett 570: 26-31, 2014.

CARRASQUILLO Y, GEREAU RW: Hemispheric lateralization of a molecular signal for pain modulation in the amygdala. Mol Pain 4: 24, 2008.

DA COSTA GOMEZ TM, BEHBEHANI MM: An electrophysiological characterization of the projection from the central nucleus of the amygdala to the periaqueductal gray of the rat: the role of opioid receptors. Brain Res 689: 21-31, 1995.

FARDIN V, OLIVERSAS JL, BESSON JM: A reinvestigation of the analgesic effects induced by stimulation of the periaqueductal gray matter in the rat. II. Differential characteristics of the analgesia induced by ventral and dorsal PAG stimulation. Brain Res 306: 125-139, 1984.

FIELDS HL, HEINRICHER MM, MASON P: Neurotransmitters in nociceptive modulatory circuits. Annu Rev Neurosci 14: 219-245, 1991.

GEBHART GF, SANDKÜHLER J, THALHAMMER JG, ZIMMERMANN M: Inhibition of spinal nociceptive information by stimulation in midbrain of the cat is blocked by lidocaine microinjected in nucleus raphe magnus and medullary reticular formation. J Neurophysiol 50: 1446-1459, 1983.

HEINRICHER MM, CHENG ZF, FIELDS HL: Evidence for two classes of nociceptive modulating neurons in the periaqueductal gray. J Neurosci 7: 271-278, 1987.

IKEDA R, TAKAHASHI Y, INOUE K, KATO F: NMDA receptor-independent synaptic plasticity in the central amygdala in the rat model of neuropathic pain. Pain 127: 161-172, 2007.

JI G, NEUGEBAUER V: Hemispheric lateralization of pain processing by amygdala neurons. J Neurophysiol 102: 2253-2264, 2009.

KAUPPILA T, KONTINEN VK, PERTOVAARA A: Influence of spinalization on spinal withdrawal reflex responses varies depending on the submodality of the test stimulus and the experimental pathophysiological condition in the rat. Brain Res 797: 234-242, 1998.

KOLBER BJ, MONTANA MC, CARRASQUILLO Y, XU J, HEINEMANN SF, MUGLIA LJ, GEREAU RW: Activation of metabotropic glutamate receptor 5 in the amygdala modulates pain-like behavior. $J$ Neurosci 30 : 8203-8213, 2010.

LAU BK, VAUGHAN CW: Descending modulation of pain: the GABA disinhibition hypothesis of analgesia. Curr Opin Neurobiol 29: 159-164, 2014.

LE DOUX J: The amygdala. Curr Biol 17: R868-R874, 2007.

LI W, NEUGEBAUER V: Block of NMDA and non-NMDA receptor activation results in reduced background and evoked activity of central amygdala neurons in a model of arthritic pain. Pain 110: 112-122, 2004. 
LOVICK TA: Integrated activity of cardiovascular and pain regulatory systems: role in adaptive behavioural responses. Prog Neurobiol 40: 631-644, 1993.

NEUGEBAUER V, GALHARDO V, MAIONE S, MACKEY SC: Forebrain pain mechanisms. Brain Res Rev 60: 226-242, 2009.

OKA T, TSUMORI T, YOKOTA S, YASUI Y: Neuroanatomical and neurochemical organization of projections from the central amygdaloid nucleus to the nucleus retroambiguus via the periaqueductal gray in the rat. Neurosci Res 62: 286-298, 2008.

OLIVEIRA MA, PRADO WA: Role of PAG in the antinociception evoked from the medial or central amygdala in rats. Brain Res Bull 54: 55-63, 2001.

PALAZZO E, MARABESE I, DE NOVELLIS V, ROSSI F, MAIONE S: Supraspinal metabotropic glutamate receptors: a target for pain relief and beyond. Eur J Neurosci 39: 444-454, 2014.

PAXINOS G, WATSON C: The Rat Brain in Stereotaxic Coordinates. Academic Press, New York, 1982.

RIZVI T A, ENNIS M, BEHBEHANI MM, SHIPLEY MT: Connections between the central nucleus of the amygdala and the mid brain periaqueductal gray: topography and reciprocity. J Comp Neurol 303: 121-131, 1991.

SAGALAJEV B, BOURBIA N, BELOUSHKO E, WEI H, PERTOVAARA A: Bidirectional amygdaloid control of neuropathic hypersensitivity mediated by descending serotonergic pathways acting on spinal $5-\mathrm{HT}_{3}$ and 5- $\mathrm{HT}_{1 \mathrm{~A}}$ receptors. Behav Brain Res 282: 14-24, 2015.

SAGALAJEV B, WEI H, CHEN Z, ALBAYRAK I, KOIVISTO A, PERTOVAARA A: Oxidative stress in the amygdala contributes to neuropathic pain. Neuroscience in press 2018 . doi: 10.1016/j.neuroscience.2017.12.009.

SPUZ CA, BORSZCZ GS: NMDA or non-NMDA receptor antagonism within the amygdaloid central nucleus suppresses the affective dimension of pain in rats: evidence for hemispheric synergy. J Pain 13: 328-337, 2012.

SPUZ CA, TOMASZYCKI ML, BORSZCZ GS: N-methyl-D-aspartate receptor agonism and antagonism within the amygdaloid central nucleus suppresses pain affect: differential contribution of the ventrolateral periaqueductal gray. J Pain 15: 1305-1318, 2014.

VAN BOCKSTAELE EJ, BAJIC D, PROUDFIT H, VALENTINO RJ: Topographic architecture of stress-related pathways targeting the noradrenergic locus coeruleus. Physiol Behav 73: 273-283, 2001. 